\title{
Specific Induction of Tumor Neovasculature Death by Modified Murine PPE-1 Promoter Armed with HSV-TK
}

\author{
N. Varda-Bloom ${ }^{a, b, f} \quad$ I. Hodisha, b,f A. Shaish ${ }^{a, b} \quad$ S. Greenberger ${ }^{a, b} \quad$ R. Tal ${ }^{a, b, f}$ \\ B. Feder ${ }^{\mathrm{e}}$ J. Roitelman ${ }^{b}$ E. Breitbart ${ }^{a} \quad$ L. Bangio $^{a} \quad$ I. Barshack ${ }^{a, c}$ R. Pfeffer ${ }^{d}$ \\ D. Harats ${ }^{a, f}$

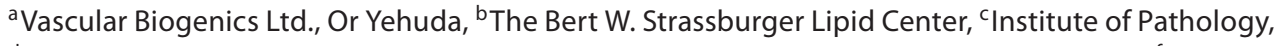

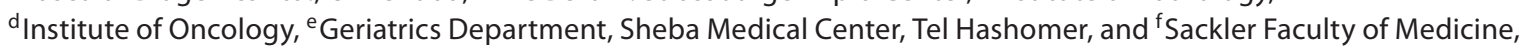 \\ Tel Aviv University, Tel Aviv, Israel
}

\section{Key Words}

Tumor angiogenesis • Antiangiogenic treatment $\cdot$ Gene therapy • Murine PPE-1 promoter · Herpes simplex virusthymidine kinase

\begin{abstract}
Background: One strategy to increase tissue specificity of gene therapy is to use promoters or enhancers. Objectives: (1) To enhance the selectivity of a murine preproendothelin-1 (PPE-1) promoter in tumor angiogenesis by using a positive endothelial transcription-binding element. (2) To test the specificity and efficiency of the modified PPE-1 promoter [PPE-1(3X)] in vitro and in vivo by using reporter genes, and the therapeutic gene herpes simplex virus-thymidine kinase (HSV-TK) in a mouse model of Lewis lung carcinoma (LLC). Results: The modified PPE-1 promoter specifically induced expression in the tumor angiogenic vascular bed with a 35-fold higher expression compared to the normal vasculare bed of the lung. Thus, when the HSV-TK gene controlled by the modified PPE-1 promoter was used systemically, it induced tumor-specific necrosis, apoptosis and mononuclear infiltrates, leading to massive destruction of the neovasculature of the pulmonary metastasis, which suppressed metastasis development. Conclusions: These results show that an
\end{abstract}

(C) 2008 S. Karger AG, Basel $1015-2008 / 08 / 0756-0346 \$ 24.50 / 0$

Fax +41613061234 E-Mail karger@karger.ch www.karger.com
Accessible online at: www.karger.com/pat adenoviral vector armed with HSV-TK controlled by the endothelial-selective murine PPE-1(3X) promoter is efficient and safe to target tumor neovasculature.

Copyright $\odot 2008$ S. Karger AG, Basel

\section{Introduction}

Specifically targeting a therapeutic gene to relevant cells is a challenging aspect of the gene therapy concept. One strategy to increase the specificity of gene therapy is to use promoters and/or enhancers that are specifically activated in the targeted cells, tissues or under defined physiological conditions [1-10]. Indeed it has been shown that specific targeting of foreign gene expression reduces transgene toxicity and prevents its expression in antigenpresenting cells, thereby decreasing the immune response against the transgene and prolonging its expression [11, 12]. In this prospective, angiogenesis, the formation of new blood vessels by sprouting of existing blood vessels, is an attractive target for gene therapy. Angiogenesis is

N.V.-B. and I.H. equally contributed to this work.
Dror Harats, MD

Vascular Biogenics Ltd., 6 Jonathan Netanyahu

IL-60376 Or Yehuda (Israel)

Tel. +972 3634 6450, Fax +97236341233

E-Mail dror@vbl.co.il 
Fig. 1. The murine PPE-1 regulatory elements inserted into the wild-type promoter to produce the PPE-1(3X). a Fragment of the regulatory element. $\mathbf{b}$ Schematic diagram of the endothelial-specific regulator cassette inserted into the wild-type murine PPE-1 promoter to generate the PPE-1(3X) promoter. The 3X fragment of the positive endothelial cell regulator containing 145 bp was inserted into the wildtype murine PPE-1 promoter downstream (-286 bp) to the endogenous fragment ( -364 to $-320 \mathrm{bp})$. The modified PPE-1 promoter, termed PPE-1(3X) (1.55 kb), was used to generate the PPE-1(3X) adenobased vectors. a GTACTTCATACTTTTCATTC CAATGGGGTGACTTTGCTTCTGG AG
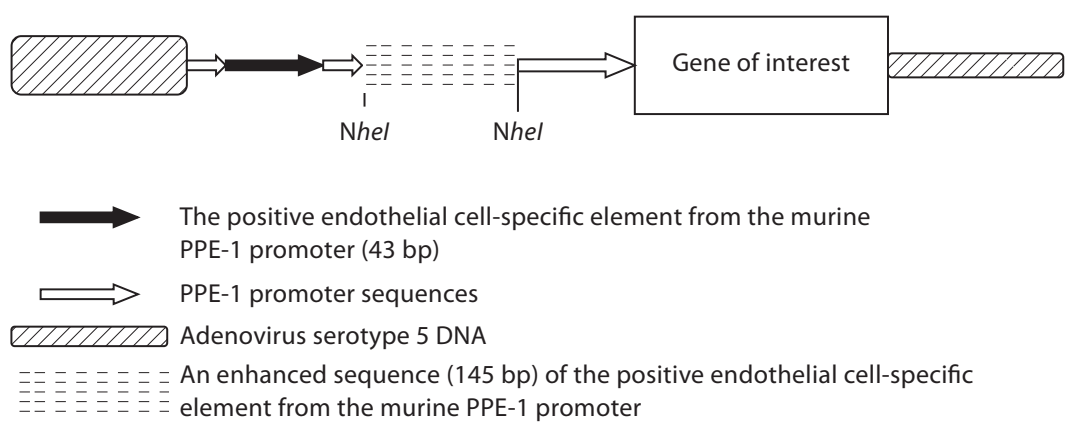

b regulated by hypoxia and growth factors and the use of gene therapy to induce antiangiogenic treatment has shown promising potential in mouse models [13-15]. We previously demonstrated [16] that the wild-type murine preproendothelin-1 (PPE-1) promoter, delivered by an adenoviral vector, leads to specific expression of the reporter genes luciferase and green fluorescence protein (GFP) in endothelial cells in vitro and in blood vessels in mouse models in vivo. However, two obstacles interfere with utilizing the wild-type murine PPE-1 promoter as an antiangiogenic-antitumor gene therapy tool: first, its relatively low levels of activity and, second, its activity in both resting and angiogenic endothelium. In the present study, we used a positive endothelial cell transcriptionbinding element, endogenously present in the murine PPE-1 promoter [17] in order to increase the promoterspecific activity in the tumor neovasculature. The specificity and efficiency of the modified PPE-1 promoter, termed PPE-1(3X) promoter, was tested in vitro and in vivo using reporter genes and the therapeutic gene herpes simplex virus thymidine kinase (HSV-TK) in the mouse model of Lewis lung carcinoma (LLC). The HSV-TK gene was used in this work as has been widely studied in gene therapy of animal tumors, providing a conditional suicide system. The enzyme phosphorylates the nucleoside analogue gancyclovir (GCV), leading to DNA polymerase inhibition, DNA replication inhibition and, consequently, cell death [16]. It was suggested that the migration of phospho-GCV to adjacent cells through gap junction augments the TK cytotoxic bystander effect [18]. To target the tumor neovasculature, Mavria et al. [19] used a retroviral vector armed with HSV-TK under the control of a hybrid ppET1-LTR promoter and showed specific killing of endothelial cells in vitro, and reduction of tumor growth in established tumors.

The PPE-1(3X) promoter showed higher efficiency (3.5-old) than the wild-type PPE-1 promoter and higher activity (35-fold) in the angiogenic tumor vasculature than in normal lung vasculature.

Moreover, systemic administration of the HSV-TK gene, under the control of the PPE-1(3X) promoter induced necrosis, apoptosis and mononuclear infiltrates and suppressed metastasis development in the LLC mod$\mathrm{el}$ and not in normal tissues. Thus suggesting that the murine PPE-1(3X) promoter is suitable for selectively targeting therapeutic genes into angiogenic tumor beds.

\section{Materials and Methods}

Modification of the Wild-Type Murine PPE-1 Promoter

The modified murine PPE-1 promoter, named PPE-1(3X), was developed by inserting extra native copies of the positive transcription element into the NheI restriction enzyme site located downstream ( $-286 \mathrm{bp}$ ) to the 43-bp endogenous positive element $(-364$ to $-320 \mathrm{bp})$. The fragment was synthesized by using two single-stranded DNA oligonucleotides (General Biotechnologies, Israel) of $96 \mathrm{bp}$ each that were annealed at the compatible sites (43 bp) followed by a fill-in reaction with Klenow fragment (MBI Fermentas, Hanover, Md., USA). The double-stranded DNA (145 bp, blunt ended) was introduced intermedially into a Bluescript plasmid (Stratagene, La Jolla, Calif., USA) in SmaI restriction sites, followed by introduction of the element into the wild-type PPE-1 promoter in the NheI restriction sites (fig. 1).

Preparation of the Recombinant Adenovirus

All recombinant type 5 adenovirus vectors used in this study were generated as previously described. In brief, GFP, luciferase and the (HSV-TK) genes were subcloned under the control of the 
PPE-1(3X) promoter by using the NotI restriction site. Adenoviruses, termed Ad5PPE-1(3X)Luc, Ad5PPE-1(3X)GFP or Ad5PPE1(3X)HSV-TK were prepared by cotransfections of HEK-293 cells with pPACPPE-1(3X)Luc/GFP/HSV-TK and the adenoviral DNA plasmid pJM17 [16].

The Ad5CMVLuc virus (kindly provided by R. Gerard from UTSw, Dallas, Tex., USA), Ad5CMV-GFP (Quantum Biotechnologies, Carlsbad, Calif., USA) and AdCMV-TK (Qbiogene Biotechnologies, Calif., USA) containing the CMV immediate early promoter were used as non-tissue-specific controls. The Ad5PPE$1 \mathrm{Luc}$ and Ad5PPE-1GFP viruses containing the wild-type murine PPE-1 promoter were previously generated in our laboratory [16] and used as controls for basal PPE-1 promoter activity.

All viruses were prepared in large scale according to the previously described procedure [20]. Viral stocks were tittered and stored at $-20^{\circ} \mathrm{C}$ at a concentration of $10^{12}$ plaque-forming units/ $\mathrm{ml}(\mathrm{pfu} / \mathrm{ml})$.

\section{Cell Culture}

LCC cells (LLC-D122-96, kindly provided by Prof. L. Eisenbach, The Weizmann Institute of Science, Rehovot, Israel), human embryonic kidney (HEK-293), HepG2 (ATCC-HB-8065) and HeLa cells were grown in high-glucose Dulbecco's Modified Eagle's Medium (DMEM) supplemented with $10 \%$ fetal calf serum (FCS), L-glutamine and $100 \mathrm{U} / \mathrm{ml}$ penicillin and streptomycin. Bovine aortic endothelial cells (BAEC, kindly provided by Prof. N. Savion, Goldshlager Institute, Sheba Medical Center, Tel Hashomer, Israel) and normal skin fibroblasts (NSF) HeLa (ATCC-CCL-2) were grown in low-glucose DMEM supplemented with $10 \%$ FCS, $200 \mathrm{mM}$ L-glutamine and $100 \mathrm{U} / \mathrm{ml}$ penicillin and streptomycin. BAEC medium was supplemented with $1 \mathrm{ng} / \mathrm{ml}$ basic fibroblast growth factor (bFGF). Human umbilical endothelial cells (HUVEC) were produced as previously described by Jaffe et al. [21] and were grown in RPMI-1640 supplemented with $10 \%$ FCS.

Luciferase Activity and GFP Expression in Cells and Tissues

Luciferase activity and GFP expression in vitro and in vivo were determined as described previously $[16,22]$.

To assay the PPE-1 promoter activity quantitatively in vitro and in vivo, we used the luciferase gene expression system kit. $48 \mathrm{~h}$ after transfection or transduction, the cells were washed and $200 \mu \mathrm{l}$ lysis buffer was added for $15 \mathrm{~min}$. Cells lysates were collected and centrifuged for $15 \mathrm{~min}(14,000 \mathrm{rpm})$ at $4^{\circ} \mathrm{C}$, followed by addition of $10 \mu \mathrm{l}$ of the supernatants to $50 \mu$ l luciferase assay buffer. The activity was measured in a luminometer over a 20 second period.

To assay luciferase activity in mice organs, tissue $(20 \mathrm{mg})$ was excised and homogenized in $1 \mathrm{ml}$ of the homogenization solution and centrifuged for $15 \mathrm{~min}(14,000 \mathrm{rpm})$ at $4^{\circ} \mathrm{C} ; 10 \mathrm{ml}$ of the supernatant were assayed for luciferase activity as described above. Results were expressed as luciferase light units per $1 \mu \mathrm{g}$ protein for cells or $1 \mathrm{mg}$ protein for tissues. Protein was measured using the Bradford assay with bovine serum albumin (BSA) as a standard.

To test the GFP expression in vitro, cells were washed twice with PBS and fixed for $30 \mathrm{~min}$ with freshly made $4 \%$ paraformaldehyde in PBS, followed by examination in a fluorescence microscope.
To test the cellular distribution of the delivered gene in vivo, tissues were fixed in freshly made $4 \%$ paraformaldehyde in $0.1 \mathrm{M}$ phosphate buffer for $6 \mathrm{~h}$ at $4^{\circ} \mathrm{C}$, soaked overnight in $30 \%$ sucrose at $4{ }^{\circ} \mathrm{C}$ and frozen in OCT compound (Sakura, USA). The tissue blocks were sliced with a cryostat at $10 \mu \mathrm{m}$ thickness and observed directly in a fluorescence microscope (fluorescein isothiocyanate filter).

The whole protocol was performed under dim light to prevent GFP bleaching.

\section{In vitro Experiments: DNA Transduction}

Cells were plated in $16-\mathrm{mm}$ dishes $24 \mathrm{~h}$ prior to transduction. DNA transduction of BAEC, HUVEC, HepG2, HeLa and NSF cells was performed by incubation with 1, 5 and 10 m.o.i (multiplicity of infection) of Ad5PPE-1(3X)Luc for $4 \mathrm{~h}$ in a total volume of $200 \mu \mathrm{l}$ growing medium, followed by incubation with the growing medium in a total volume of $2 \mathrm{ml}$ for $48 \mathrm{~h}$. Ad5PPE-1Luc was used as a control for the basal promoter activity and Ad5CMVLuc as a non-tissue-specific control.

\section{Animals}

Fourteen-week-old male C57BL/6 mice (Harlan Farms, Jerusalem, Israel) were used. All animal procedures were approved by the Animal Care and Use Committee, Sheba Medical Center, Tel Hashomer, Israel.

\section{Tissue Gene Expression in Normal Mice}

To localize the cellular expression, Ad5PPE-1-3X-GFP, Ad5PPE-1GFP or Ad5CMVGFP $\left(10^{10} \mathrm{pfu} / \mathrm{ml}\right.$ in $100 \mu \mathrm{l}$ physiologic saline) was intravenously injected into the mice ( $n=12$ in each group). GFP expression was detected by fluorescence microscopy 5 days after injection. All mice appeared healthy and no toxicity or inflammation was noted in the liver or other tissue.

\section{Tumor Implantation and Metastasis Formation}

Implantation of LLC cells was performed as previously described [16]. LLC cells, $5 \times 10^{5}$ cells $/ \mathrm{ml}$ in $50 \mu$ l physiologic saline, were injected into the footpad. When the primary tumor achieved a diameter of $7 \mathrm{~mm}$, the mice were anesthetized followed by amputation of the limb distal segment. Lung metastases developed within 3 weeks after primary tumor removal.

\section{Viral Vector Injections and GCV Treatment}

In the reporter gene experimental groups, viral vectors that contain the reporter genes GFP or luciferase (Ad5PPE-1(3X)Luc, Ad5PPE-1Luc, Ad5PPE-1(3X)GFP, Ad5PPE-1GFP, Ad5CMVLuc or Ad5CMVGFP) ( $\mathrm{n}=12$ in each group) were intravenously injected 10 days following leg amputation. In the therapeutic gene experimental groups, Ad5PPE-1(3X)HSV-TK, Ad5CMV-TK (100 $\mu \mathrm{l}$ of $10^{11} \mathrm{pfu} / \mathrm{ml}$ ) or $100 \mu \mathrm{l}$ saline were intravenously injected 5 days after leg amputation $(n=77)$. During the following 14 days, the mice received daily intraperitoneal injections of $100 \mathrm{mg} / \mathrm{kg}$ GCV according to the different treatment groups: Ad5PPE-1(3X)TK plus GCV, Ad5CMV-TK plus GCV, Ad5PPE-1(3X)-TK without GCV, and saline plus GCV. The mice were sacrificed 24 days after vector injection; $25 \%$ mice of the control group died.

Tissue Analysis

Frozen and paraffin-embedded sections of lungs and livers were stained with hematoxylin and eosin (HE). Immunostaining 
of angiogenic endothelial cells was performed on frozen sections using rat antimouse CD31 antibody (Pharmingen BD Bioscience, Franklin Lakes, N. J., USA) [23]. For apoptotic cell determination, TUNEL staining was performed on paraffin-embedded slides, cut into $4-\mu \mathrm{m}$ slices, using the in situ Cell Apoptotic Detection kit (Boehringer Mannheim, Mannheim, Germany). Anti-caspase-3 staining was performed on paraffin-embedded specimens, cut into $4-\mu \mathrm{m}$ slices, using anti-caspase- 3 polyclonal antibody (Biocare Medical, Concord, Calif., USA). Nuclei were stained with Hoechst 33258 (Sigma Diagnostic). Blood was collected from the orbital sinus and tested for liver and kidney functions.

\section{Statistical Analysis}

ANOVA for parametric variables and the Kruskal-Wallis test for nonparametric variables were used to compare more than two groups in in vitro and in vivo experiments. Student's t test for parametric variables and the Mann-Whitney test for nonparametric variables were used to compare two unpaired groups in in vitro and in vivo experiments. The Tukey multiple comparison test was performed if the ANOVA was found positive.

\section{Results}

\section{Generating the PPE-1(3X) Promoter}

A 145-bp fragment (termed 3X-fragment) composed of extra copies of the PPE-1 endothelial cell-specific transcription element binding site [17] (fig. 1a) was constructed and inserted into the wild-type murine PPE-1 promoter as described in Materials and Methods (fig. 1b). The PPE-1(3X) was sequenced and aligned with the wildtype murine PPE-1 promoter region (Genebank accession No. U07982; GI:560542).

The PPE-1(3X) Promoter Delivered by Adenoviral

Vector Efficiently and Specifically Activates Gene

Expression in Endothelial Cells in vitro

PPE-1(3X) promoter activity measured as luciferase activity was up to 450 times higher $(26,320 \pm 2,675)$ in endothelial cells (HUVEC) compared to its activity in nonendothelial cells: $152.5 \pm 3.5,58.0 \pm 4.7,313.4 \pm$ 59.1 in HeLa, HepG2 and NSF, respectively (fig. 2A). Moreover, its activity in HUVEC was 2.5 times higher than that of the wild-type PPE-1 promoter and 100 times higher than that of the CMV promoter. In nonendothelial cell lines, CMV promoter activity was much higher than that of both PPE-1X and PPE-1(3X).

To study the specific activity of PPE-1(3X) in endothelial cells, GFP expression under the control of the PPE-1(3X) promoter was compared to GFP expression under the control of CMV (fig. 2B). High expression of GFP controlled by PPE-1(3X) was detected in BAEC and no expression was detected in non-endothelial cell lines.
In contrast, no expression was detected in endothelial cell lines under the regulation of the CMV promoter while high expression of GFP was detected in the nonendothelial cells under the regulation of CMV promoter.

\section{The PPE-1(3X) Promoter Is Minimally Active in}

Normal Tissues of C57BL/6 Mice

Minimal luciferase expression was found in both highly vascularized and nonvascularized tissues of Ad5PPE-1(3X)Luc-injected C57BL/6 mice (data not shown). GFP expression detected in the mouse livers injected with Ad5PPE-1 or Ad5PPE-1(3X) viral vectors was minimal and restricted to the liver blood vessels' endothelial cells (fig. 3a). In contrast, GFP expression detected in mice injected with Ad5CMVGFP was found in the hepatocytes and no expression was detected in the blood vessels (fig. 3b).

\section{PPE-1(3X) Promoter Is Highly Active in LLC}

Metastasized Blood Vessels

To study the PPE-1(3X) activity in LLC, luciferase expression controlled by PPE-1(3X) was measured following AdPPE-1(3X) injection to mice bearing LLC lung metastases. Luciferase expression controlled by PPE-1(3X) promoter increased 35-fold in the metastasized lungs compared to its expression in normal lungs and 3.5-fold compared to its expression under the control of PPE-1 $(\mathrm{p}<0.001)$ (fig. 4). The relative luciferase expression in the metastasis lung compared to the expression in the liver was 30,000-fold higher in Ad5PPE-1(3X)Luc-treated mice. In order to localize the foreign gene expression under the regulation of PPE-1(3X) promoter, adenovirus containing GFP controlled by PPE-1(3X) was used. GFP expression controlled by the PPE-1(3X) promoter was expressed in blood vessels in the lung metastasis, as demonstrated by colocalization with anti-CD31 antibody (fig. 4b, c).

Gene Therapy with AdPPE-1(3X)-HSV-TK Suppressed Metastasis Development in LLC-Bearing Mice

A significant reduction in metastasis mass was found in mice treated with Ad5PPE-1(3X)-HSV-TK plus GCV, while a minimal reduction was found in mice treated with Ad5CMV-HSV-TK plus GCV. No effect was found in mice treated with saline plus GCV or Ad5PPE-1(3X)HSV-TK without GCV (fig. 5a, b).

The mean weight of lung metastases in mice treated with AdPPE-1(3X)-HSV-TK plus GCV was three times lower than that in AdPPE-1(3X)-HSV-TK without GCV 


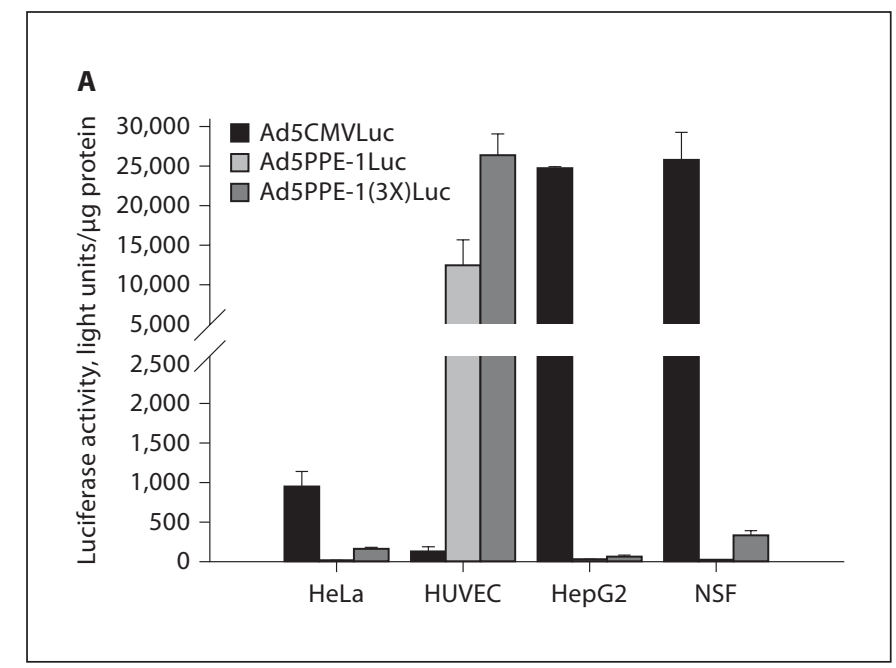

Fig. 2. The efficiency and specificity of the PPE-1(3X) adenobased vectors in vitro. A Luciferase activity in human cell lines transduced by Ad5PPE-1Luc, Ad5CMVLuc and Ad5PPE1(3X)Luc. Higher luciferase expression resulted in HeLa, HepG2 and NSF cell transduction by Ad5CMVLuc. While high luciferase expression resulted in HUVEC cell transduction by Ad5PPE1Luc or Ad5PPE-1(3X)Luc viral vectors, the luciferase expression under the control of PPE-1(3X) promoter was 2.5-25 times stronger in different experiments in endothelial cells compared to the expression under the wild-type promoter. Data represent the triplicate average of one of the three experiments performed. B GFP expression in BAEC (a), SMC (b), HeLa (c), HepG2 (d) and NSF (e) cells transduced by m.o.i. = 1 of Ad5PPE-1(3X)GFP (left column) and Ad5CMVGFP (right column). High GFP expression in BAEC (left column) resulted from Ad5PPE-1(3X)GFP transduction compared to lower GFP expression resulting from Ad5PPE1GFP transduction (data not shown), and undetectable GFP expression resulting from Ad5CMVGFP transductions (right column).

Fig. 3. GFP expression in the livers of healthy $\mathrm{C} 57 \mathrm{BL} / 6$ mice intravenously injected with Ad5PPE-1(3X)GFP (a) or Ad5CMVGFP (b). GFP under the control of PPE-1(3X) promoters was expressed in endothelial cells of the liver blood vessels. In contrast, GFP expression under the CMV promoter control was localized in the hepatocytes only and not in the blood vessels.
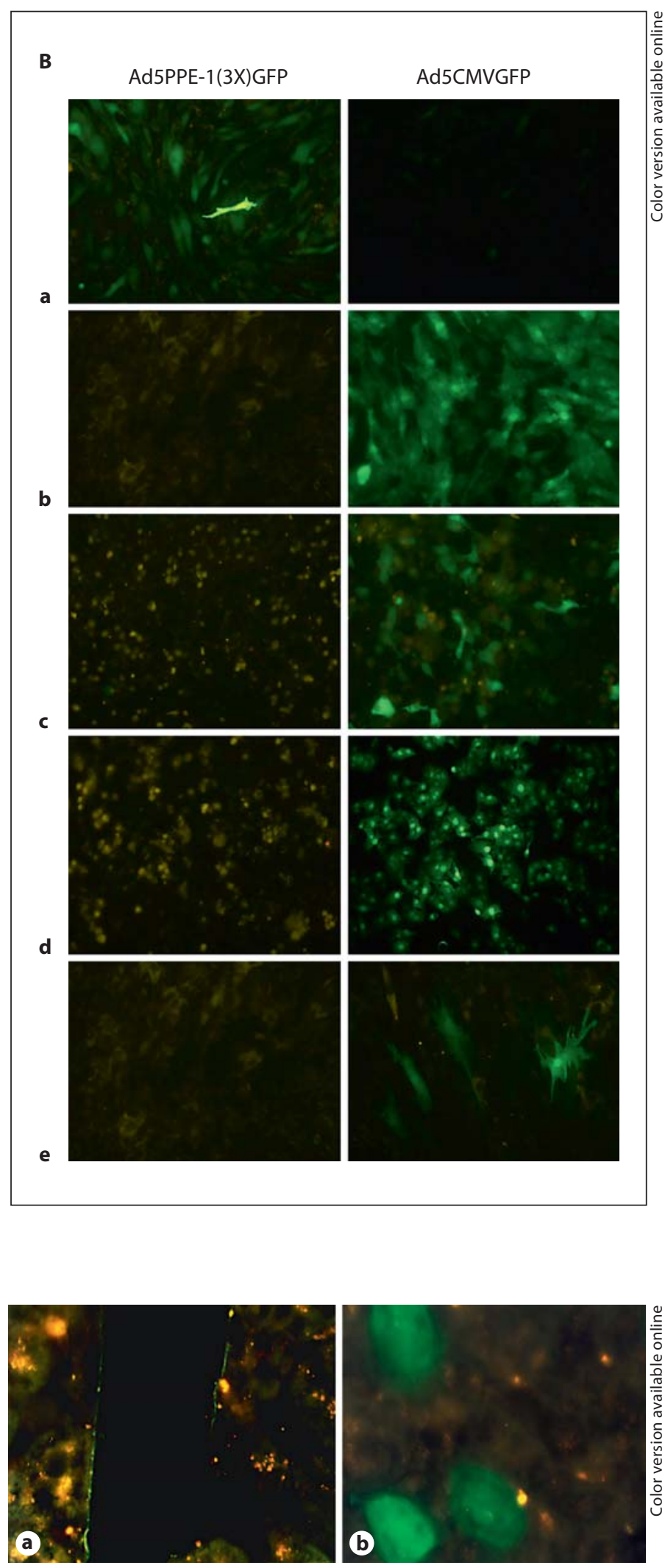

Varda-Bloom et al. 


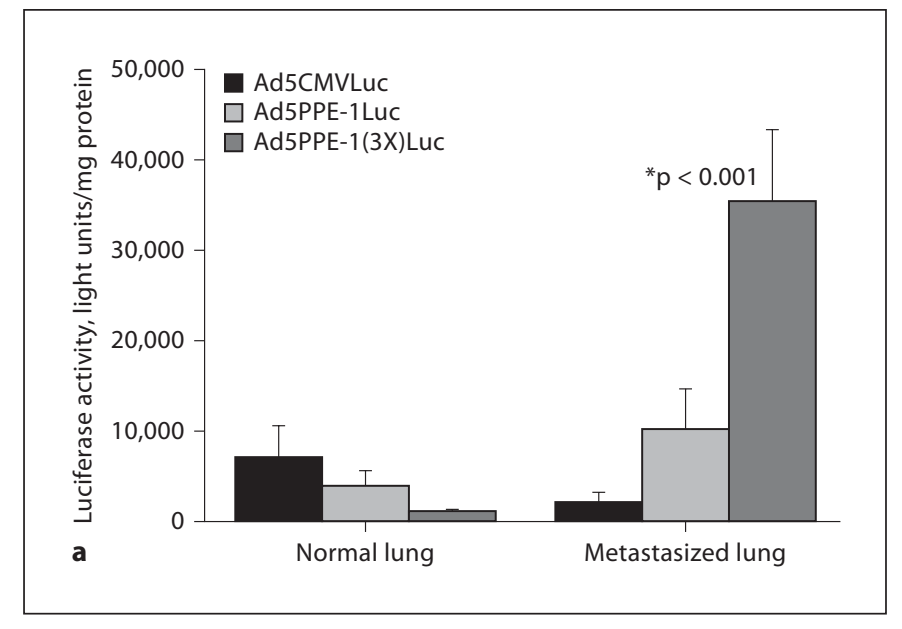

Fig. 4. Reporter gene (luciferase and GFP) expression in lung metastasis of LCC mice, injected with Ad5CMVLuc, Ad5PPE-1Luc, Ad5PPE-1(3X)Luc or Ad5PPE-1(3X)GFP. a Luciferase activity expressed as light units/mg protein was measured 5 days after systemic injection of Ad5CMVLuc, Ad5PPE-1Luc, or Ad5PPE1(3X)Luc. Luciferase expression controlled by PPE-1(3X) promot-

$(0.12 \pm 0.04$ g vs. $0.68 \pm 0.16$ g, respectively; $\mathrm{p}<0.05)$ (fig. 5b). The metastasis weight of mice treated with AdCMV-HSV-TK plus GCV was lower than that in mice treated with saline plus GCV or AdCMV-HSV-TK without GCV. However, this trend was not statistically significant.

The HSV-TK overexpression effect on the liver detected by HE staining showed mononuclear infiltrates and necrotic areas in AdCMV-HSV-TK plus GCV-treated mice but not in PPE-1(3X)-HSV-TK plus GCV-treated mice (fig. 6), indicating liver damage in the CMV-but not in the PPE-treated mice.

\section{Gene Therapy with AdPPE-1(3X)-HSV-TK Induced}

Mononuclear Infiltration, Apoptosis and Necrosis in the LLC Lung Metastases

AdPPE-1(3X)-HSV-TK plus GCV treatment induced central necrosis of the lung metastasis, while mild peripheral necrosis was detected in metastasis of the AdPPE1(3X)-HSV-TK without GCV treatment. Mononuclear infiltrates were found in Ad5PPE-1(3X)-HSV-TK plus GCV lung metastasis, while no infiltration was detected in AdPPE-1(3X)-HSV-TK without GCV treatment (fig. 6a). TUNEL staining of lung metastasis demonstrated numerous apoptotic tumor cells in AdPPE-1(3X)-HSVTK plus GCV treatment compared to few apoptotic cells following treatment with AdPPE-1(3X)-HSV-TK without GCV (fig. 6b). Anti-caspase-3 staining in lung metasta-
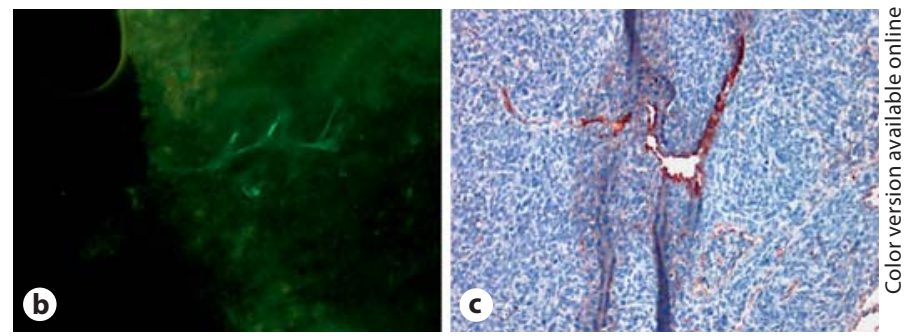

er increased 35-fold in the metastasized lungs compared to its expression in normal lungs and 3.5-fold compared to its expression under the control of PPE-1 ( $\mathrm{p}<0.001)$. b Restricted GFP expression in LLC metastasized new blood vessels. c Anti-CD31 antibody staining of new blood vessels.

sized blood vessels demonstrated massive endothelial cell apoptosis in AdPPE-1(3X)-HSV-TK plus GCV treatment compared to a small number of apoptotic cells in AdPPE1(3X)-HSV-TK without GCV (fig. 6c).

\section{AdPPE-1(3X)-HSV-TK and GCV Altered Angiogenic Vessel Morphology and Decreased Their Density in LLC Metastasis}

Anti-CD31 antibody immunohistochemistry staining revealed that the neovasculature in lung metastasis of AdPPE-1(3X)-HSV-TK plus GCV-treated mice is short and without continuity and branching. In contrast, angiogenic blood vessels from lung metastasis of AdPPE-1(3X)HSV-TK without GCV or from saline plus GCV-treated mice were long, branched and with distinctive borders (fig. 6d). Vascular density in the AdPPE-1(3X)-HSV-TK plus GCV-treated group was about 1.5 times lower than in the AdPPE-1(3X)-HSV-TK without GCV-treated group $\left(40,107 \mu \mathrm{m}^{2}\right.$ versus $61,622 \mu \mathrm{m}^{2}$, respectively).

\section{Discussion}

Transcriptional targeting is one of the strategies used to regulate therapeutic gene expression specifically in a restricted cell population or tissue and increase both the efficiency and safety of gene therapy. In the present work, we modified the murine PPE-1 promoter by inserting ex- 
Fig. 5. Effect of HSV-TK recombinant adenoviral vectors on lung metastasis development. a Representative pictures of lung metastasis in the different treatment groups indicate significant reduction of lung metastasis in AdPPE-1(3X)-TK with GCV treatment $(n=11)$ compared to AdPPE-1(3X)-TK without GCV ( $=11)$, AdCMV-TK with GCV $(\mathrm{n}=14)$ and saline with GCV treatment $(n=10)$. b Mean lung weight in the different treatment groups is demonstrated to be significantly lower in the AdPPE-1(3X)-TK with GCV treatment compared to the other treatment groups.
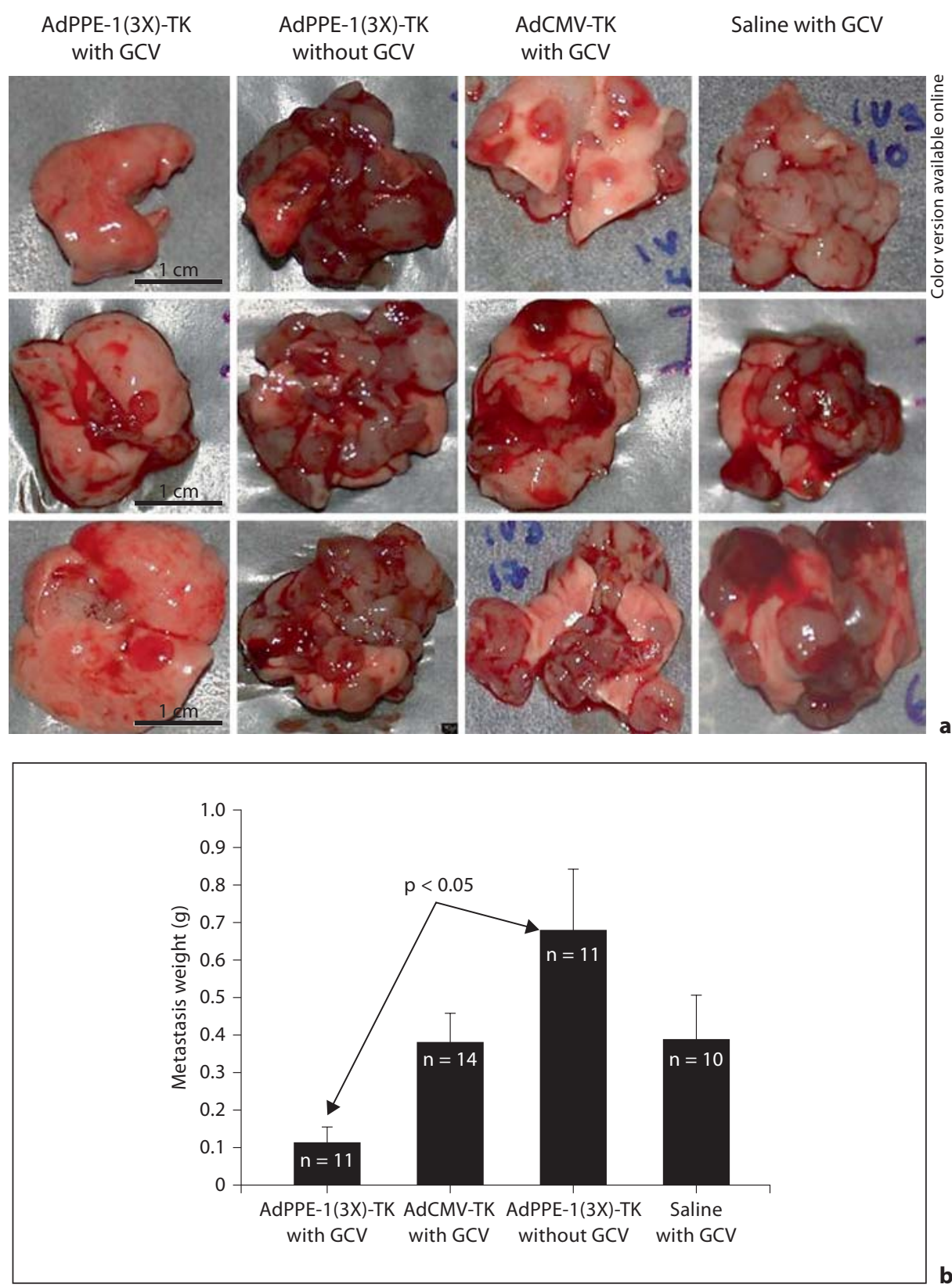

tra copies of the endothelial-specific transcription elements [17] and constructed adenovirus vectors containing GFP, luciferase or HSV-TK under the control of the modified promoter, termed PPE-1(3X). The specificity and efficiency of the promoter in tumor angiogenesis improved as a result of this modification. Moreover, overexpression of the therapeutic gene HSV-TK induced massive necrosis, apoptosis and mononuclear infiltrates, destroying the neovasculature architecture and suppressing metastasis development in the LLC mouse model.
In a previous work we used the endothelial cell-specific, wild-type PPE-1 promoter to express reporter genes in tumor angiogenesis; however, its activity in tumor angiogenesis was relatively low and was not restricted to angiogenesis and, therefore, was detected also in resting endothelium. Similar to the PPE-1 promoter, other endothelial cell-specific-promoters such as von-Willebrand factor, intracellular adhesion molecule 2 (ICAM-2), Tie 2, vascular endothelial growth factor receptor (VEGFR-2), VE-cadherin and E-selectin [24-27] were either weak or 
Fig. 6. $\mathrm{HE}$ and immunohistochemical staining of lungs excised from LLCimplanted $\mathrm{C} 57 \mathrm{BL} / 6$ mice treated with Ad5PPE-1(3X)-TK with GCV (left column) or Ad5PPE-1(3X)-TK without GCV (right column). a HE staining shows mononuclear infiltrates in lung metastasis treated with Ad5PPE-1(3X)TK and GCV, but no infiltration detected after treatment with AdPPE-1(3X)-TK without GCV. b TUNEL staining of lung metastasis demonstrated numerous apoptotic tumor cells in AdPPE-1(3X)-TK treated with GCV compared to AdPPE-1(3X)-TK without GCV. c Anti-caspase-3 staining of lung metastasized blood vessels demonstrated massive endothelial cell apoptosis in AdPPE-1(3X)-TK treated with GCV compared to AdPPE-1(3X)-TK without GCV. d Anti-CD31 staining of lung metastasis showed short blood vessels without continuity and borders in the mice treated with AdPPE-1(3X)-TK and GCV, compared to numerous blood vessels with distinctive borders and branching found after AdPPE1(3X)-TK injection without GCV.

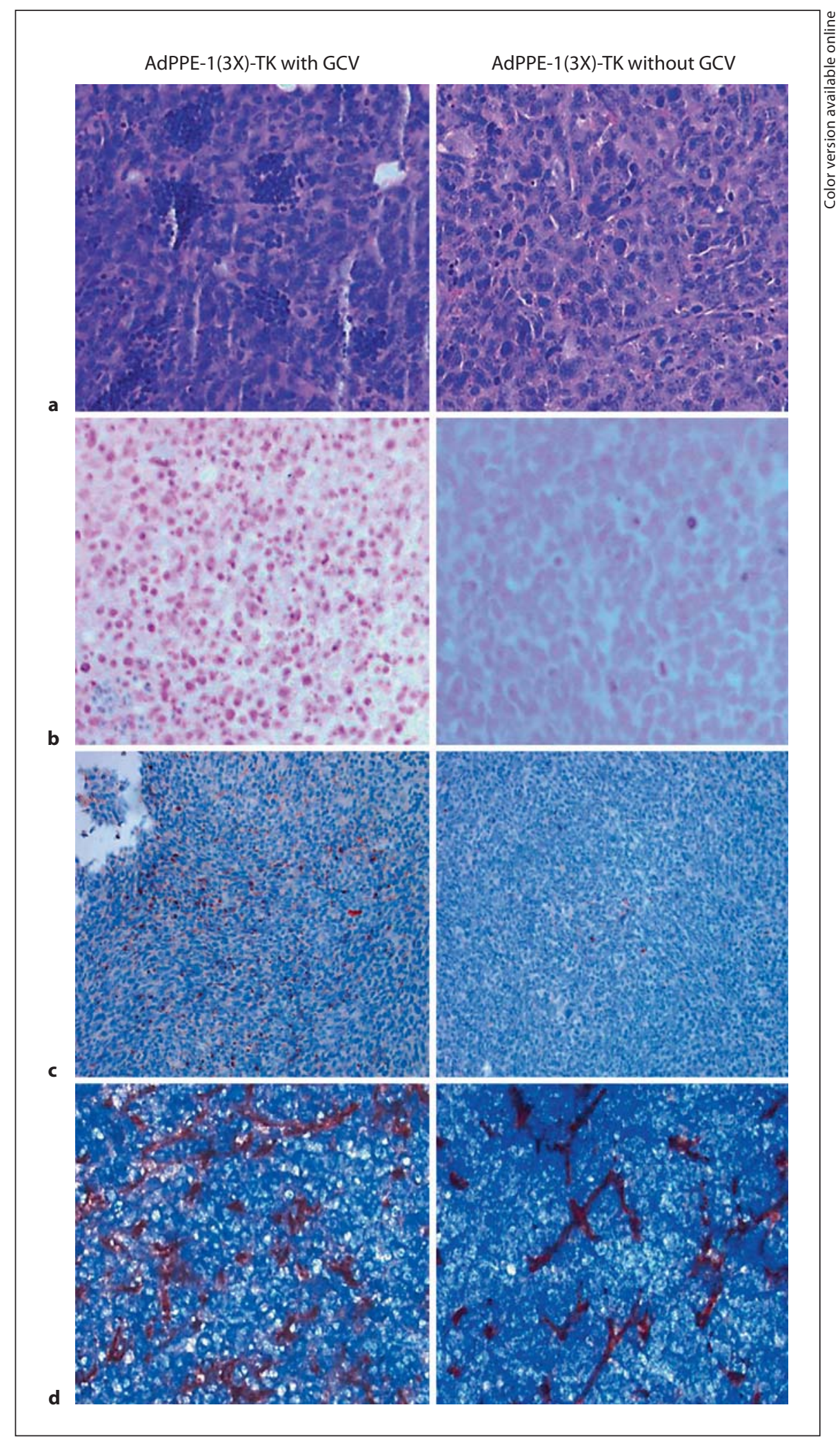


lack the specificity for tumor angiogenesis and, for that reason, systemic administration of gene therapy vectors containing these promoters was not efficient or safe. In a recent study [25], the human VEGFR-2 was used for transcriptional targeting of a viral vector to angiogenesis. Activation of caspase- 9 by the human VEGFR-2 disrupted angiogenic blood vessels in vivo. However, the viral vectors were injected into the endothelial cell implant in the mouse model, but systemic administration has not yet been studied.

In order to increase the specific activity of the PPE-1 in tumor angiogenesis, we inserted $145 \mathrm{bp}$ as $3 \mathrm{X}$ of endothelial cell-specific transcription element [17] into the wild-type murine PPE-1 promoter. This modification led to increased promoter activity in tumor-angiogenic blood vessels in Lewis lung metastasis in mice but not in the resting endothelium. This system of using the PPE-1(3X) promoter to control therapeutic gene expression selectively in neovasculature beds was proved in other angiogenesis models by using different sets of anti- or proangiogenic genes [28, 29].

In the present work, we combined the angiogenesisspecific promoter with the well-known HSV-TK/GCV system in order to induce toxicity in tumor angiogenesis. Since the toxic effect is dependent on GCV administra- tion, the cytotoxic effect in this system can be externally controlled. The AdCMV-HSV-TK showed liver toxicity, resulting in necrosis and mononuclear infiltrates, indicative of immune response induction (fig. 6). In contrast, the TK-restricted expression in the lung metastasis of Ad5PPE-1(3X)-HSV-TK-treated mice protected their livers from toxicity. Hence, using the PPE-1(3X), which is restrictively active in endothelial cells that are poor antigen-presenting cells [12], may protect against liver damage and prolong the therapeutic effect. Indeed, we found that PPE-1(3X)HSV-TK plus GCV treatment led to tissue necrosis, apoptosis and significant reduction in metastasis mass without causing any side effects due to the nonspecific expression (fig. 5, 6).

In summary, our results show the selectivity of the PPE(3X) system against tumor angiogenesis and demonstrate that HSV-TK enables a relatively safe and efficient treatment in the LLC model.

\section{Acknowledgement}

I.H., S.G., R.T., E.B., L.B., D.H. are employees of, and N.V.B., I.B., A.S. are consultants for Vascular Biogenics Ltd., a biotechnology company developing pro and antiangiogenic gene therapy products.

\section{References}

- 1 Binley K, Iqball S, Kingsman A, Kingsman S, Naylor S: An adenoviral vector regulated by hypoxia for the treatment of ischemic disease and cancer. Gene Ther 1999;6:17211727.

-2 Graulich W, Nettelbeck DM, Ficher D, Kissel T, Muller R: Cell type specificity of the human endoglin promoter. Gene 1999;227:5562.

-3 Jaggar RT, Chan HY, Harris AL, Bicknell R: Endothelial cell specific expression of tumor necrosis factor alpha from KDR or E-selectin promoters following retroviral delivery. Hum Gene Ther 1997;8:2239-2247.

4 Jäger U, Zhao Y, Porter CD: Endothelial cellspecific transcriptional targeting from a hybrid long terminal repeat retrovirus vector containing human prepro-endothelin-1 promoter sequences. J Virol 1999;73:97029709.

5 Modlich U, Pugh CW, Bicknell R: Increasing endothelial cell specific expression by the use of heterologous hypoxic and cytokineinducible enhancers. Gene Ther 2000;7:896902 .
-6 Nettelbeck DM, Jerome V, Muller R: Gene therapy: designer promoters for tumor targeting. Trends Genet 2000;16:174-181.

-7 Reynolds PN, Nicklin SA, Kaliberova L, Boatman BG, Grizzle WE, Balyasnikova K, Baker AH, Danilov SM, Curiel DT: Combined transductional and transcriptional targeting improves the specificity of transgene expression in vivo. Nat Biotech 2001; 19 : 838-842.

$\checkmark 8$ Ribault S, Nueville P, Mechine-Neuville A, Auge F, Parlakian A, Gabbiani G, Paulin D, Calenda Y: Chimeric smooth muscle specific enhancer/promoters. Valuable tools for adenovirus mediated cardiovascular gene therapy. Circ Res 2001;88:468-475.

-9 Ruan H, Su H, Hu L, Lamborn KR, Kan YW, Deen DF: A hypoxia regulated adeno-associated virus vector for cancer specific gene therapy. Neoplasia 2001;3:255-263.

10 Shibata T, Giaccia AJ, Brown JM: Development of a hypoxia responsive vector for tumor specific gene therapy. Gene Ther 2000; 7:493-498
11 Weeratna RD, Wu T, Efler SM, Zhang L, Davis HL: Designing gene therapy vectors: avoiding immune responses by using tissuespecific promoters. Gene Ther 2001;8:18721878 .

12 Mercier S, Gahery-Segard H, Monteil M, Lengagne R, Guillet JR, Eliot M, Denesvre C: Distinct roles of adenovirus vector-transduced dendritic cells, myoblasts, and endothelial cells in mediating an immune response against transgene product. J Virol 2002;76:2899-2911.

13 Sauter BV, Martinet O, Zhang WJ, Mandeli J, Woo SL: Adenovirus-mediated gene transfer of endostatin in vivo results in high levels of transgene expression and inhibition of tumor growth and metastases. Proc Natl Acad Sci USA 2000;97:4802-4807.

14 Chen CT, Lin J, Li Q,, Phipps SS, Jkubczak JL, Stewart DA, Skripchenko Y, Forry-Schaudies S, Wood J, Schnell C, Hallenbeck PL: Antiangiogenic gene therapy for cancer via systemic administration of adenoviral vectors expressing secretable endostatin. Hum Gen Ther 2000;11:1983-1996. 
15 Feldman AL, Restifo NP, Alexander HR, Barlett DL, Hwu P, Seth P, Libutti SK: Antiangiogenic gene therapy of cancer utilizing a recombinant adenovirus to elevate systemic endostatin levels in mice. Cancer Res 2000; 60:1503-1506.

16 Varda-Bloom N, Shaish A, Gonen A, Levanon K, Greenberger S, Ferber S, Levkovitz H, Castel D, Goldberg I, Afek A, Kopolovitc Y, Harats D: Tissue-specific gene therapy directed to tumor angiogenesis. Gene Ther 2001;8:819-827.

$17 \mathrm{Bu}$ X, Quertermous T: Identification of endothelin-1 cell specific regulatory region in the murine endothelin-1 gene. J Biol Chem 1997;272:32613-32622.

18 van Dillen IJ, et al: Influence of the bystander effect on HSV-tk/GCV gene therapy. A review. Curr Gene Ther 2002;2:307-322.

-19 Mavria G, Harrington KJ, Marshall CJ, Porter CD: In-vivo efficacy of HSV-TK transcriptionaly targeted to the tumor vasculature is augmented by combination with cytotoxic chemotherapy. J Gene Med 2005;7: 263-275.
20 Seijffers R, Ben David O, Cohen Y, Karasik A, Berezin M, Newgard CB, Ferber S: Increase in PDX-1 levels suppresses insulin gene expression in RIN 1046-38 cells. Endocrinology 1999;140:3311-3317.

21 Jaffe EA, Nachman RL, Becker CG, Minick CR: Culture of human endothelial cells derived from umbilical veins. Identification by morphologic and immunologic criteria. J Clin Invest 1973;52:2745-2756.

22 Harats D, Kurihara H, Belloni P, Oakley H, Ziobe A, Ackley D, Cain G, Kurihara Y, Lawn R, Sigal E: Targeting gene expression to the vascular wall in transgenic mice using the murine preproendothelin-1 promoter. J Clin Invest 1995;95:1335-1344.

-23 Ellis LM, Staley CA, Liu W, Fleming RY, Parikh NU, Bucana CD, Gallick GE: Downregulation of vascular endothelial growth factor in a human colon carcinoma cell line transfected with an antisense expression vector specific for c-src. J Biol Chem 1998; 273:1052-1057.

24 Nicklin SA, et al: Analysis of cell specific promoters for viral gene therapy targeted at the vascular endothelium. Hypertension 2001;38:65-70.

-25 Song W, Sun Q, Dong Z, Spencer DM, Numez G, Nor JE: Antiangiogenic gene therapy: disruption of neovascular networks mediated by inducible caspase- 9 delivered with transcriptionally targeted adenoviral vector. Gene Ther 2005;12:320-329.
26 Dancer A, Julian S, Bouillot S, Pointu H, Vernet M, Huber P: Expression of thymidine kinase driven by an endothelial specific promoter inhibits tumor growth of Lewis lung carcinoma cells in transgenic mice. Gene Ther 2003;10:1170-1178.

27 Walton T, Wang JL, Ribas A, Barsky SH, Economou J, Ngnyen M: Endothelium-specific expression of an E-selectin promoter recombinant adenoviral vector. Anticancer Res 1998;18:1357-1360.

28 Greenberger S, Shaish A, Varda-Bloom N, Levanon K, Breitbart E, Goldberg I, Barshack I, Hodish I, Yaccov N, Bangio L, Goncharov T, Wallach D, Harats D: Transcription controlled gene therapy against tumor angiogenesis. J Clin Invest 2004;113:10171024.

29 Levanon K, Varda-Bloom N, Greenberg S, Barshack I, Goldberg I, Orenstein A, Breitbart E, Shaish A, Harats D: Vascular wall maturation and prolonged angiogenic effect by endothelial-specific platelet-derived growth factor expression. Pathobiology 2006;73:149-158. 